\title{
Efeito da calagem na produção de arroz, feijão, milho e soja em solo de cerrado(1)
}

\begin{abstract}
Nand Kumar Fageria ${ }^{(2)}$
Resumo - A calagem é reconhecida como prática eficiente na produção das culturas nos solos ácidos dos cerrados, mas poucos são os dados de pesquisa no uso de calagem em sistema de rotação das culturas anuais. Este trabalho foi conduzido no campo durante quatro anos consecutivos (1995/96 a 1998/1999), com o objetivo de determinar os níveis adequados de calcário na produção de arroz (Oryza sativa L.) de terras altas, feijão (Phaseolus vulgaris L.), milho (Zea mays L.) e soja (Glycine max [L.] Merr.) cultivados em sucessão em um Latossolo Vermelho-Escuro distrófico de cerrado. Os tratamentos, dispostos em blocos completos ao acaso com três repetições, constaram de $0,4,8,12,16$ e $20 \mathrm{t} \mathrm{ha}^{-1}$ de calcário. As produções de feijão, milho e soja aumentaram significativamente com a aplicação de calcário, mas não houve resposta do arroz à sua aplicação. Um rendimento equivalente a $90 \%$ da produção máxima, considerado o nível econômico, foi obtido com a aplicação de 5,8 e $9 \mathrm{t} \mathrm{ha}^{-1}$ de calcário no feijão, milho e soja, respectivamente. A aplicação de calcário aumentou significativamente o $\mathrm{pH}$, os teores de $\mathrm{Ca}$ e $\mathrm{Mg}$ trocáveis, a relação $\mathrm{Ca} / \mathrm{K}, \mathrm{Ca} / \mathrm{Mg}$, a saturação por $\mathrm{Ca}$ e a saturação por $\mathrm{Mg}$ nas profundidades de $0-20$ e $20-40 \mathrm{~cm}$ no solo.
\end{abstract}

Termos para indexação: pH do solo, calcário, rotação de culturas, rendimento de grãos.

\section{Effect of liming on upland rice, common bean, corn, and soybean production in cerrado soil}

Abstract - Liming is one of the most efficient cultural practice to improve crop yields in acid soils, but there are a few data about liming in rotation system of annual crops. A field experiment was conducted for four consecutive years with the objective to determine adequate level of lime for upland rice (Oryza sativa L.), common bean (Phaseolus vulgaris L.), corn (Zea mays L.), and soybean (Glycine max [L.] Merr.) grown in rotation on a Dark-Red Latosol. Treatments arranged in a complete block design, with three replications, were the following lime rates: $0,4,8,12,16$, and 20 ton ha ${ }^{-1}$. Grain yields of common bean, corn, and soybean were significantly increased with liming. However, upland rice yield was not affected. The $90 \%$ maximum yield considered as economic level was obtained with the application of 5, 8 and 9 ton ha ${ }^{-1}$ lime on common bean, corn and soybean respectively. Soil $\mathrm{pH}$, exchangeable $\mathrm{Ca}$ and $\mathrm{Mg}, \mathrm{Ca} / \mathrm{K}$ and $\mathrm{Ca} / \mathrm{Mg}$ ratio, $\mathrm{Ca}$ saturation and $\mathrm{Mg}$ saturation increased significantly with lime aplication at $0-20$ and $20-40 \mathrm{~cm}$ soil depth

Index terms: soil $\mathrm{pH}$, limestone, rotational cropping, grain yield.

\section{Introdução}

A acidez do solo é um dos fatores que mais limitam a produtividade das culturas em várias partes do mundo, inclusive no Brasil. Nos solos ácidos existem problemas de deficiência e/ou toxidez nutricional, baixa capacidade de retenção de água e

\footnotetext{
(1) Aceito para publicação em 23 de fevereiro de 2001.

(2) Embrapa-Centro Nacional de Pesquisa de Arroz e Feijão, Caixa Postal 179, CEP 75375-000 Santo Antônio de Goiás, GO Bolsista do CNPq. E-mail: fageria@cnpaf.embrapa.br
}

baixa atividade dos microrganismos. Os solos de cerrado, com essas características, apresentam baixa produtividade em estado natural (Fageria \& Stone, 1999; Silveira et al., 2000). Para incorporação desses solos ao processo produtivo é indispensável o uso adequado de corretivos, como calcário e adubação. A calagem ainda é uma das práticas menos dispendiosas e efetivas na correção da acidez do solo, e no Brasil existem vastas reservas de calcário distribuídas em todo o território nacional.

Os solos podem ser naturalmente ácidos pela própria constituição do material de origem, como é o 
caso dos solos de cerrado, que têm baixo teor de cátions básicos, ou podem tornar-se ácidos, nas regiões em que a precipitação pluvial é maior que a evapotranspiração, responsável pela lixiviação de bases, no perfil (Fageria \& Gheyi, 1999). Com isto, as cargas positivas são substituídas por $\mathrm{Al}^{3+} \mathrm{e} \mathrm{H}^{+}$, para manutenção da eletroneutralidade (Raij, 1991). A calagem eleva o $\mathrm{pH}$ e a saturação por base do solo e fornece os nutrientes $\mathrm{Ca}$ e $\mathrm{Mg}$. A elevação do $\mathrm{pH}$ tem influência direta na redução da toxidez de Al e pode alterar a disponibilidade de nutrientes no solo para as plantas (Azevedo et al., 1996; Miranda \& Miranda, 2000).

Fageria (2000) concluiu que a cultura de arroz é bastante tolerante à acidez do solo. Porém, Barbosa Filho \& Silva $(1994,2000)$ relataram que a aplicação de $3 \mathrm{t} \mathrm{ha}^{-1}$ aumentou significativamente a produção de feijão em solo de cerrado. Da mesma forma, Oliveira et al. (1997) mostraram efeito positivo da aplicação de calcário na produção de milho em um Latossolo Vermelho-Escuro, cuja produtividade máxima foi obtida com a aplicação $6,6 \mathrm{t}$ ha-1 de calcário. Sousa et al. (1989) constataram aumentos na produção de grãos de soja com a aplicação de calcário; que a produção máxima foi obtida com $50 \%$ de saturação por bases. Embora muitas pesquisas tenham sido realizadas com calagem nas culturas anuais em solos de cerrado, os dados de pesquisa sobre calagem em sistema de rotação das culturas são escassos.

O objetivo deste trabalho foi determinar as doses adequadas de calcário para as culturas de terras altas, de arroz, feijoeiro, milho e soja cultivados em sucessão, em solo de cerrado.

\section{Material e Métodos}

Foi conduzido um experimento de campo durante quatro anos consecutivos (1995/96 a 1998/1999) em um Latossolo Vermelho-Escuro distrófico (Oxissolo) na Fazenda Capivara, da Embrapa-Centro Nacional de Pesquisa de Arroz e Feijão, localizada no Município de Santo Antônio de Goiás, GO. Os resultados da análise química das amostras do solo $(0-20 \mathrm{~cm})$ da área experimental coletadas antes da instalação do experimento, foram: $\mathrm{pH}$ em água de 5,3;10 $\mathrm{mmol}_{\mathrm{c}} \mathrm{dm}^{-3} \mathrm{de} \mathrm{Ca}^{2+} ; 10 \mathrm{mmol}_{\mathrm{c}} \mathrm{dm}^{-3}$ de $\mathrm{Mg}^{2+} ; 1,0 \mathrm{mmol}_{\mathrm{c}} \mathrm{dm}^{-3} \mathrm{de}^{3+} ; 2,6 \mathrm{mg} \mathrm{dm}^{-3}$ de $\mathrm{P}$; $121 \mathrm{mg} \mathrm{dm}^{-3} \mathrm{de} \mathrm{K}^{+} ; 4,4 \mathrm{mg} \mathrm{dm}^{-3}$ de $\mathrm{Cu} ; 1,5 \mathrm{mg} \mathrm{dm}^{-3}$ de
$\mathrm{Zn} ; 53 \mathrm{mg} \mathrm{dm}^{-3} \mathrm{de} \mathrm{Fe} ; 46 \mathrm{mg} \mathrm{dm}^{-3}$ de Mn, e $16 \mathrm{~g} \mathrm{dm}^{-3}$ de matéria orgânica. A análise granulométrica apresentou $330 \mathrm{~g} \mathrm{~kg}^{-1}$ de argila; $220 \mathrm{~g} \mathrm{~kg}^{-1}$ de silte e $450 \mathrm{mg} \mathrm{kg}^{-1}$ de areia. As análises química e granulométrica foram realizadas conforme Embrapa (1997).

O calcário foi aplicado nas doses de $0,4,8,12,16$ e $20 \mathrm{t} \mathrm{ha}^{-1}$ e incorporado com grade, aos 30 dias antes do plantio do arroz (primeiro cultivo). O calcário utilizado possuía $69,2 \%$ de PRNT; $31,45 \%$ de $\mathrm{CaO}$ e $11,6 \%$ de $\mathrm{MgO}$. A cultura de arroz recebeu $30 \mathrm{~kg} \mathrm{ha}^{-1} \mathrm{de} \mathrm{N}, 100 \mathrm{~kg} \mathrm{ha}^{-1} \mathrm{de}$ $\mathrm{P}_{2} \mathrm{O}_{5}, 60 \mathrm{~kg} \mathrm{ha}^{-1}$ de $\mathrm{K}_{2} \mathrm{O}$ e $1,5 \mathrm{~kg} \mathrm{ha}^{-1}$ de $\mathrm{Zn}$, no plantio, e $40 \mathrm{~kg} \mathrm{ha}^{-1}$ de N, em cobertura, por ocasião da iniciação do primórdio floral. A cultura do feijão, recebeu $30 \mathrm{~kg} \mathrm{ha}^{-1}$ de $\mathrm{N}, 120 \mathrm{~kg} \mathrm{ha}^{-1}$ de $\mathrm{P}_{2} \mathrm{O}_{5}$ e $60 \mathrm{~kg} \mathrm{ha}^{-1}$ de $\mathrm{K}_{2} \mathrm{O}$, e mais $40 \mathrm{~kg} \mathrm{ha}^{-1} \mathrm{de} \mathrm{N}$ em cobertura, uma semana antes do início da floração. A cultura de milho recebeu $40 \mathrm{~kg} \mathrm{ha}^{-1} \mathrm{de} \mathrm{N}$, $120 \mathrm{~kg} \mathrm{ha}^{-1}$ de $\mathrm{P}_{2} \mathrm{O}_{5}$ e $60 \mathrm{~kg} \mathrm{ha}^{-1}$ de $\mathrm{K}_{2} \mathrm{O}$ no plantio, e $40 \mathrm{~kg} \mathrm{ha}^{-1} \mathrm{de} \mathrm{N}$ em cobertura, 33 e 60 dias após o plantio. $\mathrm{Na}$ cultura de soja foram aplicados $10 \mathrm{~kg} \mathrm{ha}^{-1}$ de $\mathrm{N}$, $120 \mathrm{~kg} \mathrm{ha}^{-1} \mathrm{de}_{2} \mathrm{O}_{5}$ e $80 \mathrm{~kg} \mathrm{ha}^{-1}$ de $\mathrm{K}_{2} \mathrm{O}$ no plantio. Todas as quatro culturas receberam a mesma adubação, do mesmo modo, no segundo cultivo. O N, o P, o K e o $\mathrm{Zn}$ foram aplicados nas formas de sulfato de amônio, superfosfato triplo, cloreto de potássio e sulfato de zinco, respectivamente. As sementes de soja foram inoculadas com rizóbio Bradyrhizobium japonicum.

O delineamento experimental foi o de blocos ao acaso, com três repetições. O primeiro cultivo foi de arroz na época das águas (1995/1996), o segundo, de feijão (1996 inverno), o terceiro foi de milho nas água (1996/1997) e o quarto, de soja (1997 - inverno). As cultivares plantadas foram Caiapó, de arroz, Aporé, de feijão, Cargil 435, de milho, e EMBRAPA 20 (Doko-RC), de soja. O espaçamento entre fileiras para as culturas de arroz, feijão e soja foi de $40 \mathrm{~cm}$, e para a cultura do milho, de $80 \mathrm{~cm}$. A densidade de sementes foi de 70 por metro linear para arroz, 18 para o feijão, 25 para a soja e sete para o milho. O tamanho da parcela foi de $6 \times 5 \mathrm{~m}$, com bordadura de $1 \mathrm{~m}$ entre cada parcela e $2 \mathrm{~m}$ entre cada bloco. Colheramse as seis fileiras centrais com $5 \mathrm{~m}$ cada de arroz; as sete fileiras centrais com $4 \mathrm{~m}$ de feijão; as três fileiras centrais de $5 \mathrm{~m}$ de milho; e as seis fileiras centrais de $5 \mathrm{~m}$ de soja. Após cada colheita foram coletadas nas profundidades de 0-20 e 20-40 cm, 40 subamostras por parcela para formarem uma amostra composta. As análises químicas das amostras compostas foram realizadas conforme Embrapa (1997).

Os dados foram submetidos à análise de variância, e foi ajustada equação de regressão apropriada para determinar os efeitos dos tratamentos. 


\section{Resultados e Discussão}

A interação entre anos de cultivos e saturação por base não foi significativa $(\mathrm{P}<0,05)$ para a produção de matéria seca e produção de grãos. Portanto, apenas os dados médios relativos aos dois cultivos de cada cultura foram apresentados (Figura 1). A produção de grãos de arroz não foi influenciada pelos tratamentos, por ser uma cultura considerada bastante tolerante à acidez do solo em comparação com outras, como feijão, milho, soja e trigo (Fageria et al., 1997, 1999). Fageria \& Santos (1998) mostraram que a produção de arroz aumentou linearmente com o aumento de 0 a $38,3 \mathrm{mmol}_{\mathrm{c}} \mathrm{dm}^{-3} \mathrm{de}^{3 l^{3+}}$ no solo. Fageria \& Zimmermann (1998) também relataram a diminuição na produção de arroz de terras altas com o aumento do pH acima de 5,3 em Latossolo Vermelho-Escuro do cerrado.

A produção de feijão $(\mathrm{P}<0,01)$, milho $(\mathrm{P}<0,01) \mathrm{e}$ soja $(\mathrm{P}<0,05)$ aumentou significativamente e de forma quadrática com a aplicação de calcário. O nível econômico ( $90 \%$ da produção máxima) foi obtido com 5,8 e 9 t ha $^{-1}$ de calcário em relação às culturas do feijoeiro, milho e soja, respectivamente. A produção do feijoeiro aumentou em $21 \%$ em relação à testemunha, com aplicação de $5 \mathrm{t} \mathrm{ha}^{-1}$ de calcário. Da mesma maneira, ocorreu um aumento de $23 \%$ na produção do milho, com a aplicação de $8 \mathrm{tha}^{-1}$ de calcário, e de $31 \%$ no caso da soja, com a aplicação de $9 \mathrm{t} \mathrm{ha}^{-1}$ de calcário, em comparação com o tratamento-testemunha. Isto significa que a tolerância das culturas à acidez do solo obedeceu a seguinte ordem: arroz $>$ feijão $>$ milho $>$ soja. Fageria \& Stone (1999) relataram a tolerância dessas culturas à acidez quando cultivadas em solo de cerrado, na mesma ordem. O feijoeiro, o milho e a soja têm apresentado respostas acentuadas à calagem, com aumento significativo de produtividade, em solo de cerrado (Miranda et al., 1980; Quaggio et al., 1982; Fageria \& Stone, 1999; Caires et al., 2000). Raij \& Quaggio (1997) determinaram que as doses econômicas de calcário nos solos de cerrado para as culturas do milho, soja e feijão foram 9, 5,5 e 6,5 $\mathrm{t} \mathrm{ha}^{-1}$, respectivamente. Essas doses são semelhantes às obtidas no presente trabalho.

A aplicação do calcário acarretou aumento significativo do $\mathrm{pH}$, dos teores de $\mathrm{Ca}$ e $\mathrm{Mg}$, da relação
$\mathrm{Ca} / \mathrm{K}, \mathrm{Ca} / \mathrm{Mg}$, da saturação por $\mathrm{Ca}$ e saturação por $\mathrm{Mg}$ (Tabelas 1 e 2). Os valores do $\mathrm{pH}$ e os teores de $\mathrm{Ca}$ e $\mathrm{Mg}$ tanto na camada superficial $(0-20 \mathrm{~cm})$ como na camada subsuperficial $(20-40 \mathrm{~cm})$ são adequados para as culturas anuais como feijão, milho e soja (Fageria \& Stone, 1999). O aumento do $\mathrm{pH}$ e dos teores de $\mathrm{Ca}$ e $\mathrm{Mg}$ na camada 20-40 cm significa que houve lixiviação dos cátions $\mathrm{Ca}$ e $\mathrm{Mg}$ para essa profundidade, principalmente nas doses mais altas de calcário. Fageria et al. (1991) também relataram lixiviação dos cátions $\mathrm{Ca}$ e $\mathrm{Mg}$ para a camada de 20-40 cm com a aplicação de 6 a 12 t ha ${ }^{-1}$ de calcário, após três anos dos cultivos de arroz e feijão, em solo do cerrado. Isto significa que o uso da dose adequada de calcário possibilita a correção da acidez da camada subsuperficial. Essa condição pode ter favorecido o crescimento do sistema radicular em maior

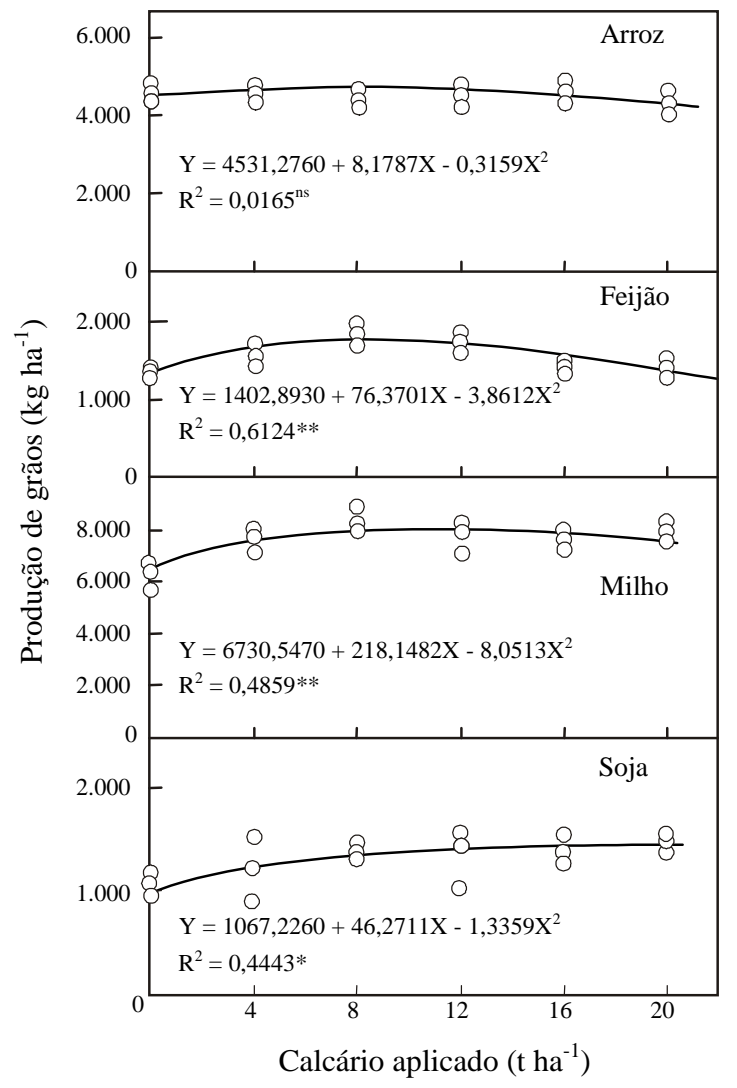

Figura 1. Relação entre dose de calcário e produção de grãos de arroz de terras altas, feijão, milho e soja, em solo de cerrado. 
Tabela 1. Valores de $\mathrm{pH}$ e teores de $\mathrm{Ca}$ e $\mathrm{Mg}$ no solo após a colheita de oito cultivos sob diferentes doses de calcário em duas profundidades.

\begin{tabular}{|c|c|c|c|c|c|c|}
\hline \multirow{2}{*}{$\begin{array}{l}\text { Dose de } \\
\text { calcário } \\
\left(\mathrm{t} \mathrm{ha}^{-1}\right)\end{array}$} & \multicolumn{3}{|c|}{$0-20 \mathrm{~cm}$} & \multicolumn{3}{|c|}{$20-40 \mathrm{~cm}$} \\
\hline & $\begin{array}{c}\mathrm{pH} \\
\text { (em água) }\end{array}$ & \multicolumn{2}{|c|}{$-\left(\mathrm{mmol}_{\mathrm{c}} \mathrm{dm}^{-3}\right)$} & $\begin{array}{c}\mathrm{pH} \\
\text { (em água) }\end{array}$ & \multicolumn{2}{|c|}{ - $\left(\mathrm{mmol}_{\mathrm{c}} \mathrm{dm}^{-3}\right)$} \\
\hline 0 & 5,6 & 19,2 & 10,9 & 5,5 & 17,6 & 9,8 \\
\hline 4 & 6,0 & 23,3 & 11,4 & 5,9 & 19,2 & 10,9 \\
\hline 8 & 6,2 & 30,0 & 12,1 & 6,1 & 23,0 & 11,1 \\
\hline 12 & 6,4 & 31,0 & 12,5 & 6,2 & 24,2 & 11,4 \\
\hline 16 & 6,5 & 33,0 & 12,5 & 6,3 & 25,7 & 11,6 \\
\hline 20 & 6,8 & 37,7 & 13,9 & 6,7 & 32,5 & 13,2 \\
\hline $\mathrm{F}$ & $* *$ & $* *$ & $\mathrm{~ns}$ & $* *$ & $* *$ & ns \\
\hline $\mathrm{CV}(\%)$ & 3 & 14 & 16 & 3 & 14 & 14 \\
\hline \multicolumn{7}{|c|}{ Regressão } \\
\hline$\beta_{0}$ & 5,7249 & 20,3928 & 10,8786 & 5,6071 & 17,0357 & 9,9369 \\
\hline$\beta_{1}$ & 5,6251 & 0,8732 & 0,1333 & 0,0493 & 0,6714 & 0,1384 \\
\hline $\mathrm{R}^{2}$ & $0,8263^{* *}$ & $0,7191 * *$ & $0,2265 *$ & $0,7814 * *$ & $0,7779 * *$ & $0,3147 * *$ \\
\hline
\end{tabular}

nsNão-significativo. ${ }^{*} \mathrm{e}^{* *}$ Significativo a $5 \%$ e a $1 \%$ de probabilidade, respectivamente.

Tabela 2. Relação $\mathrm{Ca} / \mathrm{K}, \mathrm{Ca} / \mathrm{Mg}, \mathrm{K} / \mathrm{Mg}$, saturação de $\mathrm{Ca}$, saturação de $\mathrm{Mg}$ e saturação de $\mathrm{K}$ após colheita de oito cultivos sob diferentes doses de calcário em duas profundidades.

\begin{tabular}{|c|c|c|c|c|c|c|}
\hline $\begin{array}{c}\text { Saturação } \\
\text { por base }(\%)\end{array}$ & $\mathrm{Ca} / \mathrm{K}$ & $\mathrm{Ca} / \mathrm{Mg}$ & $\mathrm{Mg} / \mathrm{K}$ & $\begin{array}{l}\text { Saturação } \\
\text { por Ca (\%) }\end{array}$ & $\begin{array}{c}\text { Saturação } \\
\text { por Mg (\%) }\end{array}$ & $\begin{array}{l}\text { Saturação } \\
\text { por K (\%) }\end{array}$ \\
\hline & \multicolumn{6}{|c|}{$0-20 \mathrm{~cm}$} \\
\hline 0 & 9,26 & 1,81 & 5,22 & 24 & 13 & 2,57 \\
\hline 4 & 11,91 & 2,05 & 5,80 & 29 & 14 & 2,49 \\
\hline 8 & 16,49 & 2,37 & 6,94 & 40 & 17 & 2,46 \\
\hline 12 & 15,04 & 2,31 & 6,50 & 37 & 16 & 2,46 \\
\hline 16 & 15,14 & 2,46 & 6,12 & 39 & 16 & 2,63 \\
\hline 20 & 18,41 & 2,72 & 6,76 & 49 & 18 & 2,67 \\
\hline F & $* *$ & $* *$ & $\mathrm{~ns}$ & $* *$ & $* *$ & $\mathrm{~ns}$ \\
\hline $\mathrm{CV}(\%)$ & 19 & 11 & 15 & 10 & 10 & 10 \\
\hline \multicolumn{7}{|l|}{ Regressão } \\
\hline$\beta_{0}$ & 10,4357 & 1,8714 & 5,6233 & 25,2321 & 13,7774 & 2,4833 \\
\hline$\beta_{1}$ & 0,4252 & 0,0478 & 0,0599 & 1,1868 & 0,1939 & 6,2499 \\
\hline $\mathrm{R}^{2}$ & $0,5975^{* *}$ & $0,5285^{* *}$ & $0,1616^{\mathrm{ns}}$ & $0,8226 * *$ & $0,4188 * *$ & $0,0320^{\mathrm{ns}}$ \\
\hline & \multicolumn{6}{|c|}{$20-40 \mathrm{~cm}$} \\
\hline 0 & 9,52 & 1,89 & 5,21 & 24 & 13 & 2,49 \\
\hline 4 & 10,68 & 1,78 & 6,01 & 26 & 15 & 2,44 \\
\hline 8 & 15,06 & 2,25 & 6,68 & 36 & 16 & 2,42 \\
\hline 12 & 13,47 & 2,13 & 6,37 & 32 & 15 & 2,44 \\
\hline 16 & 14,32 & 2,23 & 6,42 & 35 & 16 & 2,48 \\
\hline 20 & 17,88 & 2,49 & 7,20 & 44 & 18 & 2,49 \\
\hline $\mathrm{F}$ & $* *$ & ns & ns & $* *$ & $* *$ & ns \\
\hline $\mathrm{CV}(\%)$ & 19 & 15 & 15 & 10 & 9 & 13 \\
\hline \multicolumn{7}{|l|}{ Regressão } \\
\hline$\beta_{0}$ & 9,4571 & 1,7821 & 5,5666 & 22,7559 & 13,4702 & 2,4428 \\
\hline$\beta_{1}$ & 0,3767 & 0,0305 & 0,0762 & 0,9148 & 0,1859 & 0,0019 \\
\hline $\mathrm{R}^{2}$ & $0,5227 * *$ & $0,3469 * *$ & $0,2510 *$ & $0,7832 * *$ & $0,4774 * *$ & $0,0024^{\mathrm{ns}}$ \\
\hline
\end{tabular}

nsNão-significativo. ${ }^{*} \mathrm{e}^{* *}$ Significativo a $5 \%$ e a $1 \%$ de probabilidade, respectivamente. 
profundidade e refletido na produtividade das culturas (Miranda \& Miranda, 2000). Os resultados do presente trabalho também mostram que a aplicação de 4 a $8 \mathrm{t} \mathrm{ha}^{-1}$ de calcário permite produzir oito cultivos sucessivos em solos de cerrado.

Não existem muitos dados de pesquisa sobre níveis adequados da saturação por $\mathrm{Ca}, \mathrm{Mg}, \mathrm{K}$ e relação entre $\mathrm{Ca} / \mathrm{K}, \mathrm{Ca} / \mathrm{Mg}$ e $\mathrm{Mg} / \mathrm{K}$ na produção de arroz, feijão, milho e soja cultivados em solo de cerrado. Entretanto, Kamprath (1984) determinou que o nível adequado de saturação por Ca encontra-se na faixa de $25-30 \%$ para solos tropicais ácidos e intemperizados como os de cerrado. Segundo Camberato (1999), uma saturação de $\mathrm{Mg}$ maior que $10 \%$ é necessária para assegurar o fornecimento deste nutriente para as culturas anuais. Eckert (1987) determinou que o nível adequado de saturação por bases situa-se na faixa de $10 \%$ a $15 \%$ e a saturação por $\mathrm{K}$ na faixa de $2 \%$ a $5 \%$. Isto significa que a aplicação de 4 a 8 tha $^{-1}$ de calcário é suficiente para manter níveis adequados de saturação por $\mathrm{Ca}$ e $\mathrm{Mg}$ por oito cultivos sucessivos. De acordo com dados da relação de níveis adequados de $\mathrm{Ca} / \mathrm{K}, \mathrm{Ca} / \mathrm{Mg}$ e $\mathrm{Mg} / \mathrm{K}$, os valores obtidos neste trabalho, na faixa de 4 a $8 \mathrm{t} \mathrm{ha}^{-1}$ de calcário, podem ser considerados como adequados.

\section{Conclusões}

1. As doses de 5, 8 e 9 t ha ${ }^{-1}$ de calcário são suficientes para a obtenção de $90 \%$ da produtividade máxima em relação às médias de dois cultivos de feijão, milho e soja em sucessão, respectivamente.

2. A cultura de arroz de terras altas não responde à aplicação de calcário.

3. A aplicação de calcário aumenta os valores de pH em água, $\mathrm{Ca}, \mathrm{Mg}$, saturação por $\mathrm{Ca}$ e $\mathrm{Mg}$, relação $\mathrm{Ca} / \mathrm{K}$ e $\mathrm{Ca} / \mathrm{Mg}$ até a profundidade de $40 \mathrm{~cm}$.

\section{Referências}

AZEVEDO, A. C.; KÄMPF, N.; BOHNEN, H. Alterações na dinâmica evolutiva de Latossolo Bruno pela calagem. Revista Brasileira de Ciência do Solo, Campinas, v. 20, n. 2, p. 191-198, 1996.
BARBOSA FILHO, M. P.; SILVA, O. F. da. Adubação e calagem para o feijoeiro irrigado em solo de cerrado. Pesquisa Agropecuária Brasileira, Brasília, v. 35, n. 7, p. 1317-1324, jul. 2000.

BARBOSA FILHO, M. P.; SILVA, O. F. da. Aspectos agroeconômicos da calagem e da adubação nas culturas de arroz e feijão irrigado por aspersão. Pesquisa Agropecuária Brasileira, Brasília, v. 29, n. 11, p. 1657 1667, nov. 1994.

CAIRES, E. F.; BANZATTO, D. A.; FONSECA, A. F. Calagem na superfície em sistema plantio direto. Revista Brasileira de Ciência do Solo, Viçosa, MG, v. 24, n. 1, p. 161-169, 2000.

CAMBERATO, J. J. Bioavailability of calcium, magnesium, and sulfur. In: SUMMER, M. E. (Ed.). Handbook of soil science. Boca Raton: CRC, 1999. p. 53-69.

ECKERT, D. J. Soil test interpretations: basic cation saturation ratios and sufficiency levels. In: BROWN, J. R. (Ed.). Soil testing: sampling, correlation, calibration, and interpretation. Madison: Soil Science Society of America, 1987. p. 53-64.

EMBRAPA. Centro Nacional de Pesquisa de Solos (Rio de Janeiro, RJ). Manual de métodos de análise de solo. 2. ed. Rio de Janeiro, 1997. 212 p. (Documentos, 1).

FAGERIA, N. K. Resposta de arroz de terras altas à correção de acidez em solo de cerrado. Pesquisa Agropecuária Brasileira, Brasília, v. 35, n. 11, p. 2303 2307, 2000.

FAGERIA, N. K.; BALIGAR, V. C.; JONES, C. A. Growth and mineral nutrition of field crops. 2 . ed. New York: M. Dekker, 1997. 624 p.

FAGERIA, N. K.; GHEYI, H. R. Efficient crop production. Campina Grande: UFPB, 1999. 548 p.

FAGERIA, N. K.; SANTOS, A.B. dos. Rice and common bean growth and nutrient concentrations as influenced by aluminum on an acid lowland soil. Journal of Plant Nutrition, New York, v. 21, n. 5, p. 903-912, 1998.

FAGERIA, N. K.; STONE, L. F. Manejo da acidez dos solos de cerrado e de várzea do Brasil. Santo Antônio de Goiás: Embrapa-CNPAF, 1999. 42 p. (Documentos, 92).

FAGERIA, N. K.; STONE, L. F.; SANTOS, A. B. dos. Maximização da eficiência de produção das culturas. Brasília: Embrapa-SCT/Embrapa-CNPAF, 1999. 294 p.

Pesq. agropec. bras., Brasília, v. 36, n. 11, p. 1419-1424, nov. 2001 
FAGERIA, N. K.; WRIGHT, R. J.; BALIGAR, V. C.; CARVALHO, J. R. P. Response of upland rice and common bean to liming on an Oxisol. In: WRIGHT, R. J.; BALIGAR, V. C.; MURRMAN, R. P. (Ed.). Plant-soil interactions at low pH. Dordrecht: Kluwer Academic, 1991. p. 519-525.

FAGERIA, N. K.; ZIMMERMANN, F. J. P. Influence of $\mathrm{pH}$ on growth and nutrient uptake by crop plants in an Oxisol. Communications in Soil Science and Plant Analysis, New York, v. 29, n. 17/18, p. 2675-2682, 1998.

KAMPRATH, E. J. Crop response to lime on soils in the tropics. In: ADAMS, F. (Ed.). Soil acidity and liming. 2. ed. Madison: American Society of Agronomy, 1984. p. 349-368.

MIRANDA, L. N.; MIELNICZUK, J.; LOBATO, E. Calagem e adubação corretiva. In: MARCHETTI, D.; MACHADO, A. D. (Coord.). Cerrado: uso e manejo. Anais... Brasília: Editerra, 1980. p. 523-591.

MIRANDA, L. N.; MIRANDA, J. C. C. de. Efeito residual do calcário na produção de milho e soja em solo Glei Pouco Húmico. Revista Brasileira de Ciência do Solo, Viçosa, MG, v. 24, n. 1, p. 209-215, 2000.

OLIVEIRA, E. L. de; PARRA, M. S.; COSTA, A. Resposta da cultura do milho, em um Latossolo Vermelho-
Escuro álico, à calagem. Revista Brasileira de Ciência do Solo, Campinas, v. 21, n. 1, p. 65-70, 1997.

QUAGGiO, J. A.; MASCARENHAS, H. A. A.; BATAGLIA, O. C. Resposta da soja à aplicação de doses crescentes de calcário em Latossolo Roxo distrófico de cerrado: II. Efeito residual. Revista Brasileira de Ciência do Solo, Campinas, v. 6, n. 2, p. 113-118, 1982.

RAIJ, B. van. Fertilidade do solo e adubação. São Paulo: Agronômica Ceres, 1991. 343 p.

RAIJ, B. van; QUAGGIO, J. A. Methods used for diagnosis and correction of soil acidity in Brazil: overview. In: INTERNATIONAL SYMPOSIUM ON PLANT-SOIL INTERACTIONS AT LOW pH, 4., 1996, Belo Horizonte. Proceedings... Campinas: Sociedade Brasileira de Ciência do Solo, 1997. p. 205-214.

SILVEIRA, P. M.; ZIMMERMANN, F. J. P.; SILVA, S. C. da; CUNHA, A. A. da. Amostragem e variabilidade espacial de características químicas de um latossolo submetido a diferentes sistema de preparo. Pesquisa Agropecuária Brasileira, Brasília, v. 35, n. 10, p. 2057-2064, out. 2000.

SOUSA, D. M. G. de; MIRANDA, L. N. de; LOBATO, E.; CASTRO, L. H. R. de. Métodos para determinar as necessidades de calagem em solos dos cerrados. Revista Brasileira de Ciência do Solo, Campinas, v. 13, n. 2, p. 193-198, 1989. 